OKHEP-98-12

\title{
Can the QCD Effective Charge Be Symmetrical in the Euclidean and Minkowskian Regions?
}

\author{
K.A. Milton ${ }^{a *}$ and I.L. Solovtsov ${ }^{b \dagger}$ \\ ${ }^{a}$ Department of Physics and Astronomy, University of Oklahoma, Norman, OK 73019 USA \\ ${ }^{b}$ Bogoliubov Laboratory of Theoretical Physics, Joint Institute for Nuclear Research, Dubna, \\ Moscow Region, 141980 Russia
}

(January 24, 2018)

\begin{abstract}
We study a possible symmetrical behavior of the effective charges defined in the Euclidean and Minkowskian regions and prove that such symmetry is inconsistent with the causality principle.
\end{abstract}

PACS Numbers: 11.10.Hi, 11.55.Fv, 12.38.Aw, 12.38.Bx

*E-mail: milton@mail.nhn.ou.edu

$\dagger^{\dagger}$-mail: solovtso@thsun1.jinr.ru 
A possible way to resolve the ghost-pole problem for the running coupling constant obtained by using the renormalization group resummation can be found by imposing KällénLehmann analyticity, which reflects a fundamental property of local quantum field theorythe principle of causality. This idea in the QCD case has been elaborated in [1]. The correct analytic properties of the running coupling give the possibility of a self-consistent definition of the effective running coupling in the timelike, Minkowskian region [2, 3]. In this note we study a possible symmetrical behavior of the effective charges defined in the spacelike and timelike domains.

The conventional renormalization group method determines the running coupling in the Euclidean region. To find a QCD parametrization of processes that are characterized by timelike momenta, such as the process of $e^{+} e^{-}$annihilation into hadrons, one has to use some special procedure of 'analytic continuation' from the Euclidean to the Minkowskian region. To this end let consider the Adler $D$-function, which corresponds to the vector quark currents. The perturbative expansion improved by the renormalization group method in the massless case has the form $(a=\alpha / 4 \pi)$

$$
D\left(Q^{2}\right) \propto 1+d_{1} \bar{a}\left(Q^{2}\right)+d_{2} \bar{a}^{2}\left(Q^{2}\right)+\cdots .
$$

The $D$-function is an analytic function in the complex $Q^{2}$ plane with a cut along the negative real axes. Defining the effective charge by

$$
D\left(Q^{2}\right) \propto 1+d_{1} \bar{a}^{\mathrm{eff}}\left(Q^{2}\right)
$$

one can see that it must possess the following spectral representation

$$
\bar{a}^{\mathrm{eff}}\left(Q^{2}\right)=\frac{1}{\pi} \int_{0}^{\infty} \frac{d \sigma}{\sigma+Q^{2}} \rho(\sigma) .
$$

The appropriate quantity to define the effective charge in the Minkowskian region (' $s$ channel') is the $R$-ratio for the process of $e^{+} e^{-}$annihilation into hadrons. The structure of the perturbative expansion for this quantity is similar to the perturbative representation for the Adler function in the Euclidean region ('t-channel') defined by Eq. (11). The functions $D\left(Q^{2}\right)$ and $R(s)$ in some sense can be called as 't-s dual' functions and, similarly to Eq. (2), one can define the effective charge in the timelike region by

$$
R(s) \propto 1+r_{1} \bar{a}_{s}^{\mathrm{eff}}(s),
$$

where the subscript ' $s$ ' means ' $s$-channel'.

There is the following connection between these effective charges in the spacelike and timelike regions:

$$
\bar{a}^{\mathrm{eff}}\left(Q^{2}\right)=Q^{2} \int_{0}^{\infty} \frac{d s}{\left(s+Q^{2}\right)^{2}} \bar{a}_{s}^{\mathrm{eff}}(s)
$$

and

$$
\bar{a}_{s}^{\mathrm{eff}}(s)=-\frac{1}{2 \pi \mathrm{i}} \int_{s-\mathrm{i} \epsilon}^{s+\mathrm{i} \epsilon} \frac{d z}{z} \bar{a}^{\mathrm{eff}}(-z) .
$$


The contour of integration in (6) lies in the region of the analyticity of the corresponding integrand.

The effective charge in the $t$-channel is defined through the spectral function $\rho(\sigma)$ by Eq. (3). The corresponding expression for the $s$-channel charge can be written down as follows

$$
\bar{a}_{s}^{\mathrm{eff}}(s)=\frac{1}{\pi} \int_{s}^{\infty} \frac{d \sigma}{\sigma} \rho(\sigma) .
$$

Nearly a quarter century ago, Schwinger proposed [4] that the Gell-Mann-Low function, or the $\beta$-function, in QED could be represented by a spectral function for the photon propagator, which has a direct physical meaning. The $\beta$-function of the $s$-channel effective coupling constant (7) is indeed proportional to the spectral density, according to Schwinger's identification

$$
\beta_{s}(s)=s \frac{d \bar{a}_{s}^{\mathrm{eff}}(s)}{d s}=-\frac{\varrho(s)}{\pi} .
$$

Defining the $\beta$-function of the $t$-channel charge (3) $\beta\left(Q^{2}\right)=Q^{2} d \bar{a}^{\text {eff }}\left(Q^{2}\right) / d Q^{2}$ we can write down the following relation between the two $\beta$-functions

$$
\beta\left(Q^{2}\right)=Q^{2} \int_{0}^{\infty} \frac{d s}{\left(s+Q^{2}\right)^{2}} \beta_{s}(s) .
$$

Thus, the general properties of the theory lead to the following properties of the $\beta$-function considered as a function of the Euclidean momentum $Q^{2}: \beta\left(Q^{2}\right)$ is an analytic function in the complex $Q^{2}$-plane with a cut along the negative real axis.

Note that we have defined the effective charge in the $s$-channel. However, an analogous analysis can be performed for the running coupling and similar relations and conclusions can be obtained for it as well. In the framework of perturbation theory, the difference between the $t$ - and $s$-channel running coupling constants appears starting from the three-loop level (these are the well-known $\pi^{2}$-terms). Therefore, $\beta\left(Q^{2}\right)=\beta_{s}\left(s=Q^{2}\right)+O$ (3-loop). It is also at the three-loop level that the $\beta$-function coefficients become renormalization-scheme dependent.

It is interesting to consider whether there exists a possible solution, which can be called an $s$ - $t$ 'self-dual solution' of Eq. (9), in which there is a symmetrical behavior of the charges for the $t$ - and $s$ - channels. In this case $\beta\left(Q^{2}\right)=\beta_{s}\left(s=Q^{2}\right)$ and we have the following integral equation

$$
\beta\left(Q^{2}\right)=Q^{2} \int_{0}^{\infty} \frac{d s}{\left(s+Q^{2}\right)^{2}} \beta(s) .
$$

It is clear that there is a "trivial" solution to Eq. (10), $\beta\left(Q^{2}\right)=$ const. Are there any other solutions? Introduce the variables $Q^{2} / \Lambda^{2}=\exp (x)$ and $s / \Lambda^{2}=\exp (y)$, and put $\phi(x)=\beta\left(Q^{2}\right)$ and $\phi(y)=\beta(s)$, so that from Eq. (10), we obtain the integral equation

$$
\phi(x)=\int_{-\infty}^{\infty} d y K(x-y) \phi(y),
$$


with the kernel

$$
K(x)=\frac{1}{4} \frac{1}{\cosh ^{2}(x / 2)}
$$

By applying the Fourier transform to Eq. (11) one finds

$$
\tilde{\phi}(p)=\tilde{K}(p) \tilde{\phi}(p),
$$

where

$$
\tilde{K}(p)=\frac{\pi p}{\sinh (\pi p)}
$$

Possible nontrivial solutions of Eq. (13) appear at the points for which $\tilde{K}(p)=1$. However, there is only one point of that sort: $p=0$. Therefore,

$$
\tilde{\phi}(p)=\operatorname{const} \cdot \delta(p)
$$

which leads to the "trivial" solution $\beta\left(Q^{2}\right)=$ const and other $s$ - $t$ self-dual solutions are absent.

Thus, behaviors of the running couplings in the spacelike and timelike regions cannot be symmetrical in any renormalization scheme. It should be stressed that to reach this conclusion we used only the properties of analyticity, which reflect the general principle of causality, and, therefore, this result can be considered as a rigorous consequence of the first principles of quantum field theory.

\section{ACKNOWLEDGMENTS}

The authors would like to thank D.V. Shirkov and O.P. Solovtsova for interest in this work. Partial support of the work by the US National Science Foundation, grant PHY9600421, by the US Department of Energy, grant DE-FG-03-98ER41066, and by the RFBR, grant 96-02-16126, is gratefully acknowledged. I.S. also thanks the high energy group of the University of Oklahoma for its warm hospitality. 


\section{REFERENCES}

[1] D. V. Shirkov and I. L. Solovtsov, Phys. Rev. Lett. 79, 1209 (1997).

[2] K. A. Milton and I. L. Solovtsov, Phys. Rev. D 54, 5295 (1997).

[3] K. A. Milton and O. P. Solovtsova, Phys. Rev. D 57, 5402 (1998).

[4] J. Schwinger, Proc. Natl. Acad. Sci. USA 71, 3024 (1974); 71, 5047 (1974). 